feedback displayed in common areas of the ward which are regularly updated. 4. Mutual Expectations between Staff and Patients: A set of expectations created in co-production with patients displayed in the communal areas of the ward to be followed by both staff and patients. 5. Gardening sessions : One of our newer change ideas during the COVID-19 pandemic was to provide a safe, socially distanced space for patients to be involved in growing and caring for the Nile Ward garden with our activities co-ordinator. 6. Optimisation of Physical Exercise : Focus on physical activity through garden fitness sessions and 1-1 fitness sessions in the gym. This was another change idea commenced during the COVID-19 pandemic. These sessions occur throughout the day with our fitness instructor and enable our patients to focus on their physical health \& fitness. 7. Improved Ward Environment : Gym equipment were upgraded and the appearance of the ward gymnasium was enhanced using quality art created in co-production with patients.

Result. There was a $43 \%$ reduction in the number of violent incidents in the ward following QI interventions. The details of the results will be depicted in pictorial form in the poster.

Conclusion. Our patients are able to recover in a safe environment with a reduced level of violence and aggression resulting in patients receiving less rapid tranquilisation and restrictive interventions. We have had fewer assaults on staff which has made our staff feel safer to work in a busy PICU. Staff feel more confident in their role through the use of the new risk assessment tool . Patients and staff alike have given positive feedback to the changes implemented in this QI project, with violence being successfully reduced by $43 \%$. We hope that our QI project can be used as an example to show how QI methodology can enable Violence Reduction within mental health services.

\section{Improving cardiometabolic health assessments and interventions at St Charles Hospital, London}

Mehtab Rahman ${ }^{\star}$ and Vernanda Julien

Central \& North West London NHS Foundation Trust

${ }^{*}$ Corresponding author.

doi: 10.1192/bjo.2021.571

Aims. St Charles is one of the largest inpatient mental health units in London with 8 wards and covers the boroughs of Kensington \& Chelsea and Westminster. This project aimed was set up so that 95\% of patients in St Charles Mental Health Centre would have a complete cardiometabolic health assessment by December 2020 . This would include Weight, Smoking, Alcohol, Substance Use, Hypertension, Cholesterol and Diabetes assessments with necessary interventions recorded. The outcome of the intervention would improve overall physical health and life expectancy.

Method. People with serious mental illness experience significantly worse physical health and shorter life expectancy of up to 10 to 15 years than the general population. CNWL is making Physical Health of patients in Mental Health Services a priority. Performance in this area has been challenging across the Trust because of:

- Buy in from clinicians.

- Staff did not feel empowered to discuss interventions with patients.

- High sickness and absence as a result of COVID was found to directly correlate with reduced physical health monitoring/ recording.

- Lack of training in completing the SystmOne physical health template
The following cardiometabolic risk monitoring interventions were recorded on SystemOne (electronic documentation platform) and performance reviewed using Tableau : Weight, Smoking, Alcohol, Substance Use, Hypertension, Cholesterol and Diabetes assessments with necessary interventions recorded.

Result. Prior to the commencement of this project, the wards in St Charles Mental Health Centre completed physical health assessments on roughly $8 \%$ of the patients in February 2020. The QI project was implemented in June 2020. By September 2020, physical health recording across 8 wards across St Charles had increased to $89 \%$ following successful implementation of the interventions.

Conclusion. The following interventions resulted in a significant improvement in physical health cardiometabolic risk monitoring at a busy inpatient mental health setting:

- Monthly physical heath meetings to enable shared learning with ward doctors, nurses and healthcare assistants.

- Ongoing one-to-one and group support to train staff with completing and recording physical health assessments.

- Tableau Physical Health Report regularly reviewed with MDT during ward round meetings.

- Physical health leads given supernumerary days to run physical health clinics on the wards.

- Fortnightly Physical health monitoring meetings with the Director of Nursing and Head of Governance.

\section{Manualising the induction of higher trainees in psychiatry for North Wales: The CiSGC Guide ("Croeso i Seiciatreg Gogledd Cymru")}

Jawad Raja ${ }^{1 *}$, Jiann Lin Loo ${ }^{1}$, Rajvinder Sambhi ${ }^{1}$ and Somashekara Shivashankar ${ }^{2}$

${ }^{1}$ Ysbyty Maelor Wrecsam, Betsi Cadwaladr University Health Board and ${ }^{2}$ Cardiff and Vale University Health Board

${ }^{*}$ Corresponding author.

doi: 10.1192/bjo.2021.572

Aims. There is a significant period of adjustment for new higher trainees in psychiatry given the presence of inter-trust differences in the National Health Services (NHS). It may take some time for a trainee to become familiar with the new administrative system and workflow of the new environment, which may be even longer for an international medical graduate (IMG). Although there is an existing induction system, having a written structured manual will assist the trainees to get through this process more easily. Hence, this Quality Improvement Project (QIP) outlined the creation of an induction manual that serves as a starter pack to facilitate the settling-in process of new North Wales higher trainees in psychiatry, i.e. the "Croeso i Seiciatreg Gogledd Cymru" (CiSGC) guide (means Welcome to North Wales Psychiatry in Welsh).

Method. The induction manual was initially drafted by the authors based on the available printed policies and information online. Further input and from different stakeholders were obtained to triangulate and enrich the manual. Specific links and further references were included in the manual for the reference of prospective manual users. Authors' contact details were included for any further clarification, suggestions or input.

Result. The manual consisted of four sections: A) General Process before, during and after Reporting Duty, B) Trainees' Duty, 3) Speciality-specific Guidance, and 4) Health Board-related Information. The General Process section covered the visa-related information, post-acceptance paperwork process, access to email 